\title{
Discordant Prolongation of the Refractory Period and Repolarization Time by a Class III Agent, E4031, in the Healing Phase of Myocardial Infarction
}

\author{
Seiji Takatsuki, MD, Hideo Mitamura, MD, \\ Koichiro Steyoshi, MD, Hideaki KanKI, MD, \\ Izumi FurunO, MD, \\ and Satoshi OGalia, MD
}

\section{SUMmarY}

Susceptibility to reentrant tachyarrhythmias and the antiarrhythmic efficacy of class III agents are related more to the duration of the refractory period (ERP) than to the repolarization time (RT). We measured both ERP and RT in a canine model of healing myocardial infarction, and evaluated the effect of a class III agent (E4031) on these parameters and on the inducibility of ventricular tachyarrhythmias. ERP and RT on the unipolar electrogram were measured at several cycle lengths in the normal (NZ) and infarct zones (IZ), respectively, in 10 canine myocardial infarction models and extrastimulation was used to induce ventricular arrhythmias. Measurements were repeated after E4031 administration.

At baseline, both ERP and RT were significantly longer in IZ than in NZ with ERP/RT ratio also higher in IZ. This ratio tended to increase at longer cycle lengths. E4031 increased ERP and RT both in NZ and IZ at all cycle lengths, but increased the ERP/RT ratio predominantly in IZ. E4031 prevented induction of sustained VT or VF, which was inducible in 3 out of 10 dogs at baseline, although it facilitated induction of VF in 1 dog with no baseline arrhythmia. By increasing the ERP/RT ratio, class III drugs may shorten the relative refractory period in IZ at the expense of a greater ERP difference created between NZ and IZ. (Jpn Heart J 1998; 39: 687-697)

Key words: Class III antiarrhythmic agent, Myocardial infarction, Ventricular arrhythmia, Refractory period, Repolarization, Canine

\footnotetext{
A FTER the CAST study, "increasing attention has been directed to class III antiarrhythmic agents which characteristically delay repolarization. However, a recent report from the SWORD trial ${ }^{2)}$ demonstrated that a pure class III

From the Cardiology Division, Department of Medicine, Keio University School of Medicine, Tokyo, Japan. Presented in part at the 17th Annual Scientific Sessions of the North American Society of Pacing and Electrophysiology (NASPE), May 1996.

Address for correspondence: Seiji Takatsuki, MD, Cardiology Division, Department of Medicine, Keio University School of Medicine, 35 Shinanomachi, Shinjuku-ku, Tokyo 160, Japan.

Received for publication March 9, 1998.

Revised and accepted June 22, 1998.
} 
agent may reduce the survival of patients after myocardial infarction. Although the precise mechanism involved has not been determined, the cause of increased mortality in this trial was most likely the proarrhythmic effect of class III agents. What remains unknown is how these agents exert anti- or pro-arrhythmic actions in the non-homogeneous milieu of the infarcted myocardium, which might differ from the homogeneous normal myocardium examined in most experimental studies.

The pharmacologic action of class III antiarrhythmic agents is to prolong repolarization, whereas susceptibility to reentrant tachyarrhythmias is more related to the duration of the refractory period than to the repolarization time, although the repolarization time is an important factor determining refractoriness. However, the relationship between the refractory period and the repolarization time may not necessarily be uniform after myocardial infarction. It is known that postrepolarization refractoriness with an effective refractory period extending beyond the action potential develops in the setting of acute myocardial ischemia. The antiarrhythmic effect of class III agents may also be influenced by the characterisitics of the substrate tissues. We therefore designed the present study to clarify the relationship between the repolarization time and the refractory period in the healing phase of myocardial infarction, and to evaluate the effect of a class III antiarrhythmic agent (E4031) on these parameters as well as on the inducibility of ventricular tachyarrhythmias.

\section{Methods}

Canine myocardial infarction model: Ten adult mongrel dogs of either sex (weighing 9.5 to $14.1 \mathrm{~kg}$ ) were anesthetized with sodium pentobarbital $(30 \mathrm{mg} / \mathrm{kg}$ intravenously), intubated with a cuffed endotracheal tube, and ventilated with room air by a constant-volume ventilator (model 80V, Harvard Apparatus, South Natick, MA, USA). Using sterile technique, thoracotomy was performed in the fifth left intercostal space and the ribs and lungs were retracted. The pericardium was then incised and the heart revealed. The left anterior descending artery was isolated and tied with silk just distal to the first diagonal branch. Five minutes before ligation, methylprednisolone $(30 \mathrm{mg} / \mathrm{kg}$ ) was administered intravenously, since this has been reported to increase the survival of a narrow rim of myocardium. ${ }^{3)}$ Thirty minutes after ligation, the chest was closed in layers, and negative pressure was reestablished in the pleural cavity. Antibiotics were administered for 5 days after surgery. This investigation conformed with the guide for the Care and Use of Laboratory Animals published by the US National Institutes of Health.

Creation of complete atrioventricular block: Seven days later, all dogs were 
again anesthetized with sodium pentobarbital, intubated, and ventilated by the Harvard respirator. The right femoral artery was cannulated with a heparinized saline-filled polyethylene catheter to monitor blood pressure continuously. The right femoral vein was cannulated with a polyethylene catheter for infusion of $0.9 \%$ saline and drug administration. Median sternotomy was performed, and the pericardium was opened and used as a cradle by suturing to the sternal wound. The body temperature was maintained at 36 to $37^{\circ} \mathrm{C}$ with a heating light. The left carotid artery was exposed, incised, and cannulated with a large tip catheter for ablation (Webster, USA). The tip of the catheter was placed at the noncoronary cusp of the aortic valve. A bioelectric amplifier (AB-600G, Nihon Koden, Tokyo, Japan) and a paper recorder (Polygraph System, Nihon Koden) were used to record His bundle electrogram from the catheter. Ablation was performed where the most prominent $\mathrm{H}$ wave was recorded, and was started with 45 Joules from a generator (CAT-500, Microbipolar Coagulator, Central Industrial Inc, Ichikawa, Japan). Once complete atrioventricular block was created, backup right ventricular pacing was applied if the ventricular rate became slower than $60 \mathrm{bpm}$. Complete AV block was created to allow pacing over a wide range of pacing intervals.

Electrophysiologic study: Two pairs of bipolar electrodes were inserted into the epicardium of the right ventricle (NZ) and the left ventricular infarct zone (IZ), and were used for simultaneous unipolar cathodal stimulation and unipolar electrogram recording in each zone. A programmable stimulator (BC-02A, Fukuda Denshi Inc, Tokyo, Japan) was used to deliver 2-msec pulses at twice diastolic threshold. The bioelectric amplifier (AB-600G, Nihon Koden) and paper recorder mentioned above were used to record the ventricular unipolar electrogram (filtered from 0.53 to $1000 \mathrm{~Hz}$ ). Lead II of the ECG was also monitored continuously.

The late diastolic excitability threshold was measured at each site and unipolar cathodal stimulation was performed at twice diastolic threshold. A train of eight stimuli (S1) was followed by a late premature stimulus (S2), and the S1S2 interval was shortened in 5-msec decrements until the effective refractory period (ERP) was reached. The ERP was defined as the longesi S1-S2 interval at which the $\mathrm{S} 2$ stimulus failed to produce a ventricular response on two successive attempts. ERPs were determined at basic pacing cycle lengths of 300, 400, 600, $800 \mathrm{msec}$ in the $\mathrm{NZ}$ and IZ.

The repolarization time (RT) was defined as the period between the onset of the stimulus artifact and the point where the end of the unipolar deflection crossed the isoelectric line in the unipolar epicardial electrogram recorded by the plunge electrode adjacent to the stimulating electrode. RT measurements were performed at the eighth beat during constant pacing at cycle lengths of 300,400 , 
600 , and $800 \mathrm{msec}$.

To induce ventricular tachyarrhythmias, unipolar cathodal stimulation with single or double ventricular extrastimuli was applied from the epicardium of the right ventricular outflow tract. Following constant ventricular pacing with an S1$\mathrm{S} 1$ interval of $300 \mathrm{msec}$, an extrastimulus was applied with the S1-S2 coupling interval decreasing by 10-msec decrements until the ERP was reached. If a sustained ventricular tachyarrhythmia was not induced, a second extrastimulus was added in a similar fashion. Ventricular tachyarrhythmia was defined as nonsustained (NSVT) if 3 or more repetitive ventricular responses were initiated but terminated spontaneously, and as sustained (SVT) if the arrhythmia persisted for $30 \mathrm{sec}$ or was accompanied by hemodynamic compromise. If the ventricular response was less than 3 nonstimulated complexes, it was classified as noninducible (NI).

These procedures were repeated after E4031 administration.

E4031 administration: E4031 was administered as a bolus injection of $50 \mu \mathrm{g} /$ $\mathrm{kg}$ followed by a maintenance infusion of $0.5 \mu \mathrm{g} / \mathrm{kg} / \mathrm{min}$. E4031 was provided by Eisai Co. Ltd. (Tokyo, Japan) and was administered in normal saline solution. Arterial blood samples for determination of the serum concentration were withdrawn at 15 minutes after the bolus injection and at the termination of the experiments. The blood was centrifuged to obtain serum, which was stored frozen. Serum E4031 concentrations were measured at Sekisui Clinical Laboratory (Tokyo).

Statistical analysis: Results are given as the mean $\pm \mathrm{SD}$. Analysis of variance was used to compare electrophysiologic characteristics over different pacing cycle lengths. For other statistical comparisons, a paired $t$-test was used. For all comparisons, $p<0.05$ was the criterion of statistical significance.

\section{Results}

Serum E4031 concentration: The serum concentration of E4031 ranged from $1.8 \pm 1.1 \mu \mathrm{g} / \mathrm{m} l$ at 15 minutes after bolus injection to $2.1 \pm 1.5 \mu \mathrm{g} / \mathrm{m} l$, at the end of the experiments. There was no significant difference between these two values and the drug concentration was considered to be sufficiently constant during the whole experiment.

Diastolic excitability threshold: The baseline diastolic excitablity threshold was significantly higher in the IZ than in the NZ $(p<0.05)$. After E4031 administration, no significant change of this threshold was observed in either the NZ or the IZ $(1.2 \pm 0.6$ to $1.7 \pm 1.2 \mathrm{~V}$ and $2.5 \pm 1.3$ to $2.3 \pm 0.8 \mathrm{~V}$, respectively).

Baseline electrophysiologic study: At 7 days after myocardial infarction, the RT was significantly longer than the ERP at all pacing cycle lengths in both left 

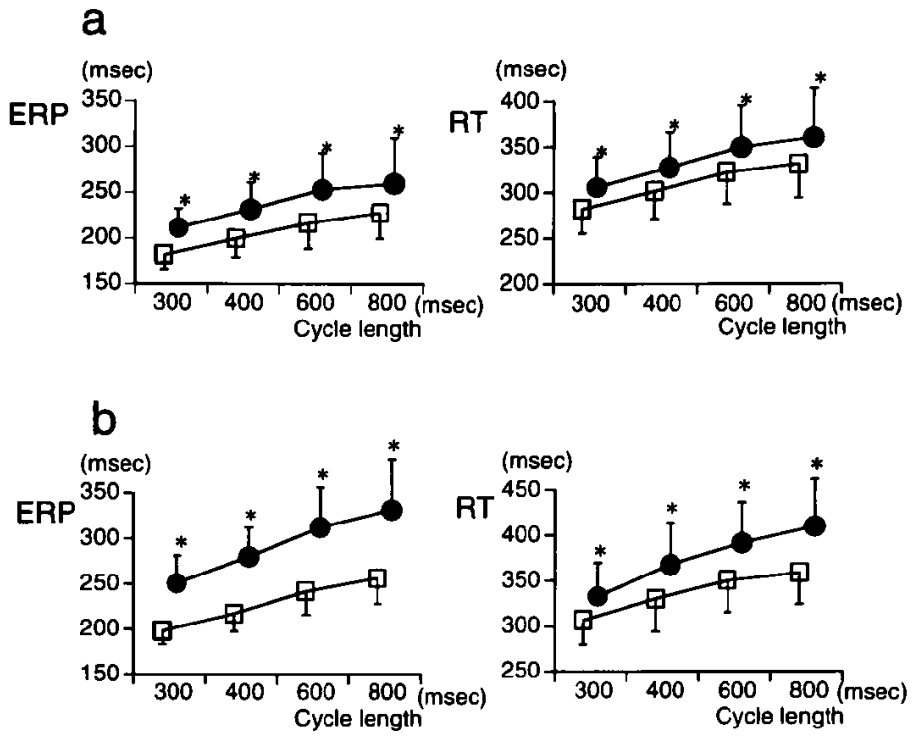

Figure 1. a: Effective refractory period (ERP) and repolarization time (RT) in the right ventricular normal zone $(\square)$ and left ventricular infarct zone at baseline. Both ERP and RT are significantly longer in the $I Z$ than the $N Z$ at every cycle length. *: $p<0.01$ vs. NZ. b: ERP and RT in the NZ ( $\square$ ) and IZ after E4031 administration. Both ERP and RT are significantly longer in the $\mathrm{IZ}$ than the $\mathrm{NZ}$ at every cycle length. The difference of ERP and RT between the two zones was increased by E4031. *: $p<0.01$ vs. NZ.

ventricular infarcted myocardium (IZ) and right ventricular normal myocardium (NZ). Both ERP and RT were significantly longer in the IZ than in the NZ at all pacing cycle lengths $(p<0.01)$ (Figure la). In both zones, ERP and RT became shorter as the pacing cycle length was decreased.

Effects of E4031: The ERP and RT values obtained during E4031 administration are shown in Figure 1b. E4031 prolonged the ERP and RT in both the NZ and IZ at all cycle lengths. However, prolongation of ERP and RT was more prominent in the IZ (Figure 2). E4031 showed a tendency to prolong ERP and RT at longer cycle lengths in each zone, but the rate dependency of its class III effect was not significant in this model (Figure 2). The ERP/RT ratio was significantly higher in the IZ at baseline $(p<0.05)$ (Figure 3 ). In the NZ, the ERP and RT were both comparably prolonged by E4031, so the ERP/RT ratio was unchanged. In contrast, ERP prolongation by E4031 was more prominent than that of $\mathrm{RT}$ in the IZ. As the result, the ERP/RT ratio increased significantly $(p<0.05)$ and also tended to increase at longer pacing cycle lengths after E4031 administration (Figure 3). As mentioned above, the diastolic excitable threshold did not change with E4031 administration, and did not affect the ERP/RT ratio among all dogs. 

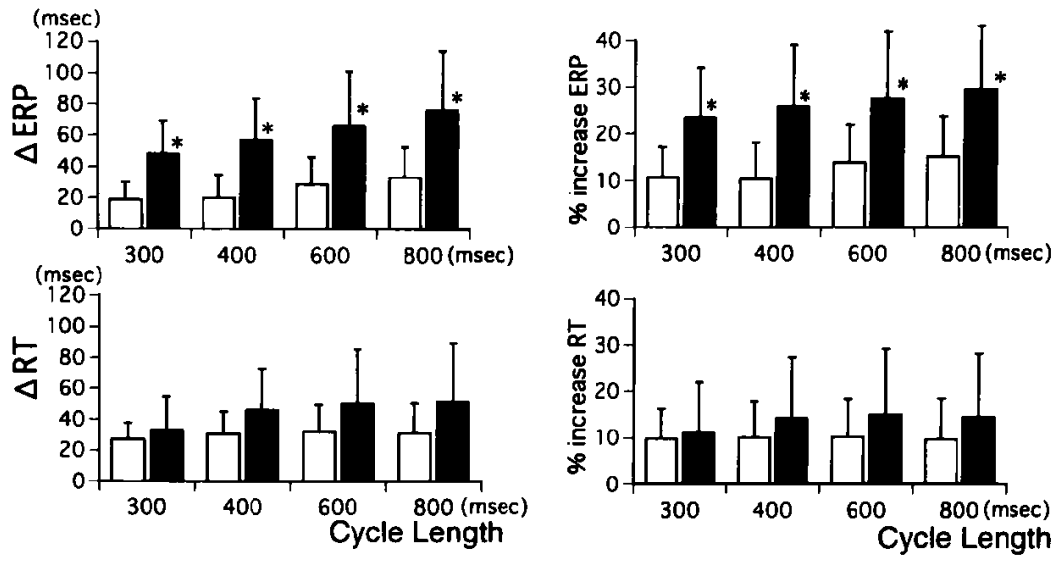

Figure 2. Prolongation of ERP $(\triangle E R P)$ and $R T(\triangle R T)$ by E4031 is shown in the left top and left bottom panels. E4031 prolonged the ERP by more in the IZ (black columns) than in the NZ (white columns) at each cycle length. RT also tended to be longer in the IZ than in the NZ. The \% Increase of ERP and RT by E4031 is shown in the right panels. There was no significant difference in the \% increase of these parameters at different pacing cycle lengths. $*: p<0.05$ vs. NZ.

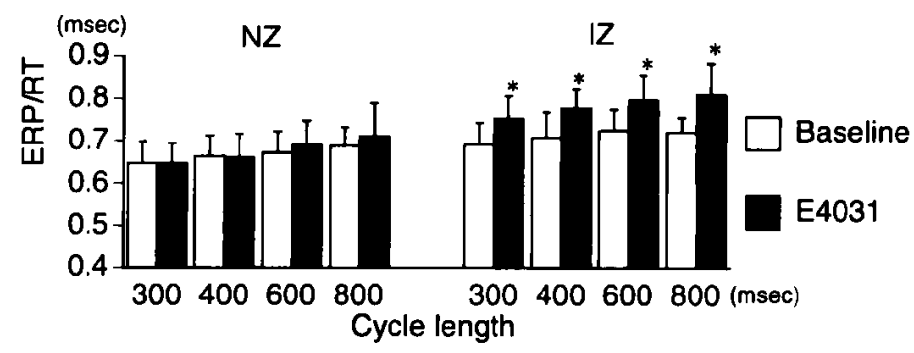

Figure 3. The ERP/RT ratio tended to increase at longer pacing cycle lengths, and was higher in the IZ than in the NZ both at baseline and after E4031 administration. E4031 did not change the ERP/RT value in the NZ, but significantly increased it in the IZ at every cycle length. *: $p<0.05$ vs. baseline.

Inducibility of ventricular arrhythmias: During the baseline study, 6 out of 10 dogs showed induction of either NSVT $(n=3)$, SVT $(n=2)$, or VF $(n=1)$. All dogs with VF or SVT induced during the baseline study showed only NSVT after E4031 administration. However, in one dog with NI at baseline, VF was induced after E4031 administration (Figure 4). In this dog, the ERP at a basic cycle length of $400 \mathrm{msec}$ was prolonged by $110 \mathrm{msec}$ in IZ compared with 20 msec in NZ after E4031 administration. Hence the ERP difference between IZ and NZ was markedly increased from $30 \mathrm{msec}$ at baseline to $120 \mathrm{msec}$ after E4031 administration, which was much greater than the mean ERP difference between IZ and NZ for all dogs $(28 \pm 16 \mathrm{msec}$ at baseline and $65 \pm 26 \mathrm{msec}$ after 


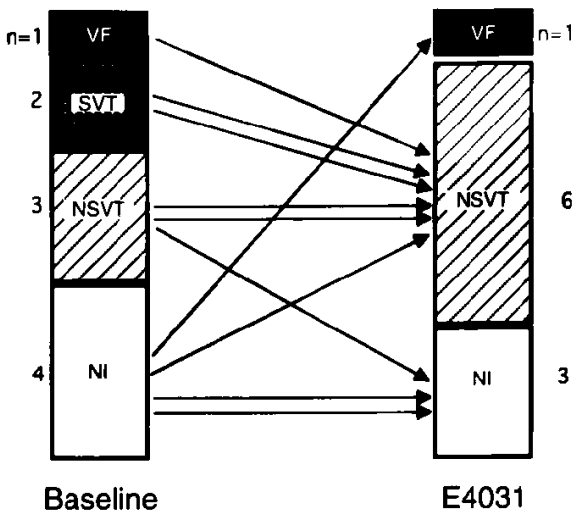

Figure 4. Inducibility of ventricular arrhythmias in postinfarction dogs before (Baseline) and after intravenous administration of E4031. Each arrow represents one animal, and the responses to programmed stimulation were categorized as non inducible (NI), nonsustained ventricular tachycardia (NSVT), sustained ventricular tachycardia (SVT), or ventricular fibrillation (VF).

E4031 administration). Thus, E4031 showed both anti- and proarrhythmic effects.

\section{Discussion}

Major findings: In dogs with 7-day-old myocardial infarction, both ERP and RT were longer in the infarct border zone than in the normal zone and the difference of ERP between the NZ and the IZ was greater than that of RT. After E4031 administration, ERP and RT prolonged both in NZ and IZ, but the degree of prolongation of both parameters was greater in the IZ than in the NZ. Furthermore, E4031 increased the ERP more than RT in the IZ, leading to a greater ERP/RT ratio. As a result, E4031 shortened the relative refractory period in the IZ at the expense of a greater ERP difference between NZ and IZ.

Electrophysiologic characteristics of the 7-day post MI canine model: The duration of the ERP varies with time after myocardial infarction. At 1 week in our canine model, ERP was longer in the IZ than in the NZ. Several other studies have shown that ERP is longer at the epicardial border of the infarcted myocardium than in the normal myocardium during the subacute phase of myocardial infarction. ${ }^{47)}$ In contrast, the action potential duration (APD) has been reported to decrease in the same phase of myocardial infarction. Ursell et al. reported that the APD at $90 \%$ repolarization of surviving epicardial muscle superfused in vitro was significantly decreased at 5 days after myocardial infarction. ${ }^{8)}$ The action potential of infarcted myocardium during this phase shows a triangular configuration, with the APD at 50\% repolarization being 
shorter than that of the normal myocardium. ${ }^{3,8)}$ However, almost no data are available concerning the APD of infarcted myocardium in vivo, probably because of technical problems. Duff et al. reported that the repolarization time of the infarct zone can be assessed by the QT interval of the surface bipolar electrogram, which was prolonged during the subacute to healing phase in a canine postmyocardial infarction model with inducible sustained ventricular tachycardia. ${ }^{97}$ In our study, RT was defined similarly to the method described by Duff et al. and is considered to reflect local epicardial repolarization, although the possibility cannot be ruled out that it may actually reflect distant electrotonicity. Preferential class III effect of E4031 in the IZ: The effects of class III antiarrhythmic agents may vary between normal and infarcted myocardium. In this study, E4031 caused greater refractory period prolongation in infarcted myocardium than in normal myocardium. Some of the experimental studies using post-infarction models have shown that class III agents prolong the refractory period to the same degree in both infarcted and normal myocardium, ${ }^{10-13)}$ while others have suggested that these agents prolong the refractory period more in infarcted myocardium. ${ }^{1-16)}$ The reason for discrepancy among studies is not clear, but may be related to methodological differences, including the presence or absence of anesthesia or thoracotomy, or the doses of the drugs used. The predominant action of E4031 on the infarcted myocardium may be related to inhomogeneous sympathetic innervation after myocardial infarction. Stanton and Zipes have pointed out that sympathetic denervation can modulate the effects of drugs in a canine myocardial infarction model. ${ }^{17)}$ It has been demonstrated that sympathetic stimulation or $\beta$-adrenergic stimulation can decrease or even cancel the effect of class III antiarrhythmic agents ${ }^{18,19)}$ on the normal myocardium because of the IKs activation. In our experimental model, the open chest condition in addition to the post-infarction status could have created a milieu where adrenergic tone was increased. Hence, it can be assumed that prolongation of the refractory period and repolarization time by E4031 was antagonized by increased sympathetic activity in the normal myocardium, whereas in the sympathetically denervated infarcted tissues the effect of E4031 was well preserved. Thus, the variable sensitivity of inhomogeneous myocardial tissue to sympathetic activity may contribute to the inconsistent electrophysiologic effects of E4031.

We should also consider the possibility of augmentation of IK blockade in the infarcted myocardium by $\mathbf{E} 4031$. When the membrane potential is depolarized to some extent, IK will decay more slowly, allowing it to play a greater role in repolarization. ${ }^{20)}$ Moreover, the drug bound to the channels will unbind more slowly at a less negative potential due to the voltage dependence of the drugchannel interaction. ${ }^{21)}$ 
Discordant prolongation of the refractory period and repolarization time in infarcted myocardium by E4031: The refractory period is defined as the duration of time in which the myocardium restores its excitability. In this study, E4031 prolonged the refractory period along with the repolarization time in normal myocardium. However, in infarcted myocardium, this drug showed discordant prolongation of the refractory period and repolarization time, with greater prolongation of the former. To our knowledge, this finding has never been described before. Although the precise mechanism involved was not clarified in the present study, there are two possibilities. The resting membrane potential is partially depolarized in the subacute phase of myocardial infarction, ${ }^{8}$ ) leading to a decrease of INa. Class III agents block IK more in the infarcted myocardium, and thus may further depolarize the resting membrane potential, leading to further decrease of $\mathrm{INa}$, which would create postrepolarization refractoriness. Although whether blocking IKr alone can depolarize the resting membrane potential has not been proved, such a hypothesis may be plausible since the resting membrane potential of the infarcted myocardium may be maintained by other currents than IK $1 .{ }^{22)}$

An alternative explanation for discordant prolongation in the IZ can be as follows. In the healing phase of myocardial infarction, the action potential duration has a triangular configuration with a short plateau followed by gradual tapering of the phase 3 segment. ${ }^{23)}$ Class III agents prolong the action potential by delaying phase 3 , and thus widen the plateau phase with subsequent steep recovery. This altered configuration of the action potential may account for the greater prolongation of the refractory period than that of the repolarization time in the IZ. Our observation that ERP/RT tended to increase at longer pacing cycle lengths may support this hypothesis. We admit however that these mechanisms remain speculative and require further basic investigations. Irrespective of the mechanism, discordant prolongation of the refractory period and repolarization time in IZ may have an antiarrhythmic effect by shortening the relative refractory period, ${ }^{24}$ thus preventing the variable conduction required for initiation of reentry.

Antiarrhythmic effect of E4031 and clinical implications: In this study, E4031 showed ambivalent pharmacologic properties, i.e., both anti- and proarrhythmic effects. E4031 may exert an antiarrhythmic action if it prolongs the refractory period preferentially in the infarcted myocardium, thus creating bidirectional block in the reentry substrate, or if it shortens the relative refractory period by increasing the ERP/RT ratio. This may be one of the mechanisms by which class III drugs suppress fatal arrhythmias, such as sustained VT or VF. On the other hand, E4031 may also exhibit a proarrhythmic action by causing early afterdepolarization during bradycardia or by increasing the difference of the 
refractory periods between normal and infarcted myocardium, as shown in our dog with VF inducible only after E4031 administration. However, the mechanisms for both anti- and pro-arrhythmic effects of this drug in each dog cannot be determined by this study alone, more detailed evaluation of dispersion of refractoriness and conduction characteristics using high resolution mapping will be required. In addition, since our observations were obtained in an experimental canine model of 7-day-old myocardial infarction with open chest and anesthesia, further studies will be required before the results can be extrapolated clinically.

Conclusions: In the healing phase of myocardial infarction, both ERP and RT were longer in the infarct border zone than in the normal zone, with the difference of ERP being greater than that of RT. These changes were further exaggerated by the administration of a class III drug, E4031. By augmenting the prolongation of these parameters and increasing the ERP/RT ratio in the IZ, E4031 can shorten the relative refractory period in the IZ at the expense of a greater ERP difference between NZ and IZ. These findings may account for the variable anti- or pro-arrhythmic actions shown by this agent.

\section{ReFERENCES}

1. Echt DS, Liebson PR, Mitchell LB, et al. the CAST Investigators. Mortality and morbidity in patients receiving encainide, flecainide, or placebo: the Cardiac Arrhythmia Suppression Trial. N Engl J Med 1991; 324: 781-8.

2. Waldo AL, Camm AJ, deRuyter $\mathbf{H}$, et al. Preliminary mortality results from the Survival with Oral dSotalol (SWORD) Trial [Abstract]. J Am Coll Cardiol 1995; 15A.

3. Scherlag BJ, Brachmann J, Kabell G, Harrison L, Guse L, Lazzara R. Sustained ventricular tachycardia: common functional properties of different anatomic substrates. In Zipes DP, Jalife J, editor. Cardiac Electrophysiology and Arrhythmias. Orlando: Grune and Stratton, 1985: 379-87.

4. Patterson E, Scherlag BJ, Berlin KD, Lazzara R. Electrophysiologic actions of BRB-I-28 in ischemically injured myocardium. J Cardiovasc Pharmacol 1993; 21: 637-46.

5. Aidonidis I, Brachmann J, Rizos I, et al. Electropharmacology of the bradycardic agents alinidine and zatebradine (UL-FS 49) in a conscious canine ventricular arrhythmia model of permanent coronary artery occlusion. Cardiovasc Drug and Ther 1995; 9: 555-63.

6. Aidonidis I, Egel E, W Kuebler, Brachmann J. Effects of prenylamine and AQ-A 39 on reentrant ventricular arrhythmias induced during the late myocardial infarction period in conscious dogs. $\mathrm{J}$ Cardiovasc Pharmacol 1993; 22: 401-7.

7. Black SC, Butterfield JL, Lucchesi BR. Protection against programmed electrical stimulation-induced ventricular tachycardia and sudden cardiac death by NE-10064, a class III actiarrhythmic drug. J Cardiovasc Pharmacol 1993; 22: 810-8.

8. Ursell PC, Gardner PI, Fenoglio J, Wit AL. Structural and electrophysiological changes in the epicardial border zone of canine myocardial infarcts during infarct healing. Circ Res 1985; 56: 436-51.

9. Duff HJ, Martin JME, Ramberg M. Time-dependent change in electrophysiological milieu after myocardial infarction in conscious dogs. Circulation 1988; 77: 209-20.

10. Lynch JJ, Hearny LA, Wallace AA, Gehret JR, Selnick HG, Stein RB. Suppression of lethal ischemic ventricular arrhythmias by the class III agent E4031 in a canine model of previous myocardium infarction. J Cardiovasc Pharmacol 1990; 15: 764-75.

11. Chi LG, Mu DX, Lucchesi BR. Electrophysiology and antiarrhythmic action of E-4031 in the experi- 
mental animal model of sudden coronary death. J Cardiovasc Pharmacol 1991; 17: 285-95.

12. Kondoh $\mathrm{K}$. Hashimoto $\mathrm{H}$, Nishiyama $\mathrm{H}$, et al. Effects of $\mathrm{MS}-5.51$, a new class III antiarrhythmic drug, on programmed stimulation-induced ventricular arrhythmia, electrophysiology, and hemodynamics in a canine myocardial infarction model. J Cardiovasc Pharmacol 1994: 23: 674-80.

13. Black SC: Butterfield JL, Lucchesi BR. Protection against programmed electrical stimulation-induced ventricular tachycardia and sudden cardiac death by NE-10064, a class III antiarrhythmic drug. J Cardiovasc Pharmacol 1993; 22: 810-8.

14. Katoh H, Ogawa S, Furuno I, Sato Y', Yoh S, Saeki K, et al. Electrophysiological effects of E-4031, a class III antiarrhythmic agent, on re-entrant ventricular arrhythmias in a canine 7-day-old myocardial infarction model. J Pharmacol Exp Ther 1990; 253: 1077-82.

15. Mitamura H, Michelson EL, Kasicwski C, Sauermeich C, Dreifus LS. Antiventricular tachyarrhythmic etlect of oral clofilium in a chronic canine myocardial infarction model. $\mathrm{J}$ Am Coll Cardiol 1984; 3: $5+2$.

16. Cobbe SM, Hoffman E, Ritzenhoff A, Brachman J, Kubler W, Senges J. Action of sotalol on potential reentrant pathways and ventricular tachyarrhythmias in conscious dogs in the late postmyocardial infarction phase. Circulation 1983; 68: 865-71.

17. Stanton MS Zipes DP. Modulation of drug effects by regional sympathetic denervation and supersensitivity: Circulation 1991; 84: 1709-14.

18. Sager PT, Follmer C, Uppal P, Pruitt $C$, Godfrey R. The effect of $\beta$-adrenergic stimulation on the frequency-dependent electrophysiologic actions of amiodarone and sematilide in humans. Circulation 1994: 90: 1811-9.

19. Sanguinetti MC; Jurkiewicz NK, Siegl PKS. Isoproterenol antagonizes prolongation of refractory period by the class III antiarrhythmic agent E-4031 in guinea pig myocytes. Circ Res 1991; 68: 77 84.

20. Furukawa T, Tsujimura $Y$, Kitamura K, Tanaka H, Habuchi Y. Time- and voltage-dependent block of the delayed $\mathrm{K}^{+}$current by quinidine in rabbit sinoatrial and atrioventricular nodes. $\mathrm{J}$ Pharmacol Exp Ther 1989; 25l: 756-63.

21. Colatsky TJ, Follmer CH, Starmer CF. Channel specificity in antiarrhythmic drug action: mechanism of potassium channel block and its role in suppressing and aggravating cardiac arrhythmias. Circulation 1990; 82: 2235-42.

22. Noma $\mathbf{A}$, Matsuda $\mathbf{H}$. The role of potassium channels in maintaining resting potential in normal and anoxic cardiac muscle. In: Escande D, Standen $\mathrm{N} s$, editor. $\mathrm{K}^{+}$Channels in Cardiovascular Medicine. Paris: Springer-Verlag France Co Inc, 1993: 41-51.

23. Wit AL, Janse MJ. Delayed ventricular arrhythmias in the subacute phase of myocardial infarction. In: The Ventricular Arrhythmias of Ischemia and Infarction. 1993; 267-356.

24. Kupersmith J. Clinical Manual of Flectrophysiology. Baltimore: Willians and Wilkins, 1993: 169-85. 